\title{
Pain after Open Preperitoneal Repair versus Lichtenstein Repair: A Randomized Trial
}

\author{
Simon Nienhuijs $\cdot$ Erik Staal $\cdot$ Mariël Keemers-Gels • \\ Camiel Rosman $\cdot$ Luc Strobbe
}

Published online: 18 May 2007

(C) Société Internationale de Chirurgie 2007

\begin{abstract}
Background The open preperitoneal approach in inguinal hernia repair might have the benefit of a mesh in the preferred space without the disadvantages of an endoscopic procedure.

Methods A total of 172 patients with primary inguinal hernia were randomized to undergo the open preperitoneal Kugel or the standard open anterior Lichtenstein procedure in a teaching hospital. The main outcome measures were operating variables, visual analog scale (VAS) pain scores, and consumed analgesics during the first 2 weeks postoperatively and at 3 months, neurological examination, and complications.

Results In the Lichtenstein group the operation took longer (54 min versus $41 \mathrm{~min} ; p<.001)$. There were no clinically important differences in VAS pain score or number of analgesics during the first 2 weeks postoperatively. In the Kugel group the mean VAS pain score at 3 months was less $(0.3$ versus $0.9 ; p=.002)$, as was the proportion of patients reporting pain (21 versus $40 \%$; $p=.007)$. Pain was merely described as neuropathic, especially in the Lichtenstein group. With the anterior repair significantly more nerves were encountered, numbness reported, and cutaneous sensory changes found with neurological examination (all $p<.001$ ).
\end{abstract}

S. Nienhuijs $(\bowtie) \cdot$ M. Keemers-Gels · C. Rosman .

L. Strobbe

Department of Surgery, Canisius-Wilhelmina Hospital,

Nijmegen, The Netherlands

e-mail: s.nienhuijs@hccnet.nl

E. Staal

University Medical Centre St. Radboud, PO Box 9015,

6500 GS, Nijmegen, The Netherlands
Conclusions For those surgeons preferring an open approach, the Kugel procedure is a feasible alternative for the standard Lichtenstein procedure and is associated with less chronic pain at three months. Most likely the neuropathic pain and numbness with the Lichtenstein technique are results of more nerves at risk with the anterior approach.

In inguinal hernia repair the endoscopic approach is associated with less postoperative pain. In two trials with 5 years of follow-up this repair was also superior in terms of chronic pain compared to open techniques $[1,2]$. Nevertheless, the open approaches are used more frequently. Reported reasons are the demands of the endoscopic technique for anesthesia, experience level, and costs. Most surgeons reserve this approach for specific indications and in dedicated centers.

There remains a need to diminish the main complication of inguinal hernia repair as chronic pain is reported in $28 \%-37 \%$ of the patients [3]. Due to the complexity of chronic pain, the true mechanism still is unclear. However, the position of a mesh is probably one factor. In some anterior techniques a (part of a) mesh is placed under the fascia transversalis, a route that passes through the inguinal canal. Furthermore, all anterior approaches require to some extent dissection of the inguinal canal, and this involves manipulation of at least one or two inguinal nerves. An open preperitoneal approach might have the benefit of a mesh in the preferred preperitoneal space without the disadvantages of an endoscopic procedure. The purpose of this trial was to compare an open preperitoneal to an open anterior approach in terms of chronic pain, as such a study has not been reported before. Therefore the technique described by Kugel [4] and the present standard technique of Lichtenstein were used. 


\section{Materials and methods}

\section{Randomization}

The study was conducted between December 2004 and September 2005 in a regional teaching hospital. The eligibility criteria were fulfilled if adult patients who had been referred for elective primary, unilateral inguinal hernia repair and did not have an irreducible inguinoscrotal hernia or previous hernia repair via the preperitoneal approach, gave informed consent. For that purpose these patients received a diary including written information about the aims and contents of the study. The regional ethics committee approved the study protocol.

A total of 172 consecutive patients were included in the study. Randomization was done using a computer generated list. In consecutive order the patients were allocated to receive one of the two repairs. The patients themselves were blinded to the method used. Patients characteristics recorded were age, sex, body mass index, ASA, and employment status (Table 1).

\section{Treatment}

Inguinal hernia repairs were performed by staff surgeons as well as surgeons in training. According to our protocol, all patients received $7.5 \mathrm{mg}$ midazolam and pre-emptive analgesics: 1,000 $\mathrm{mg}$ paracetamol and $15 \mathrm{mg}$ meloxicam, approximately $1 \mathrm{~h}$ before surgery. Prophylactic antibiotics were not used. The Lichtenstein operation was performed as described elsewhere [5]. Briefly, a $6 \times 11$-cm polypropylene mesh (Prolene; Ethicon) was trimmed to fit the inguinal floor, as necessary. The mesh was sutured to the ligament of Poupart with a non-absorbable suture and secured cranially using an absorbable suture. For the Kugel repair the open, preperitoneal approach was used as described by Kugel [4]. KugelMesh (Bard, Inc), medium oval size $(11 \times 14 \mathrm{~cm})$, was used in each case. This patch is constructed of a double layer of monofilament polypropylene with a transverse opening in the anterior layer. Hereby a pocket is created to facilitate positioning. A memory recoil ring allows the patch to maintain its shape during placement in the preperitoneal space. Further specifications and illustrations have been reported previously [4]. Nerves and vessels were spared whenever possible. In both techniques, the skin was closed with a subcuticular absorbable suture.

In the Lichtenstein group 61 patients received spinal anesthesia using 15-22.5 mg plain bupivacaine; in 4 patients complementary general anesthesia was needed due to incomplete block. The remaining 25 patients in this group preferred general anesthesia: volatile anesthetics with additional opiates (fentanyl and morphine). In the Kugel group the number of patients was 63 for spinal anesthesia with 8 complementary general anesthesia's and 23 for general anesthesia from the start. Local anesthetic $(10 \mathrm{ml}$ bupivacaine $0.75 \%$ ) was administered into the wounds of all patients. Paracetamol up to $4 \mathrm{~g}$ and meloxicam up to 15 mg were recommended as a supplementary analgesic. Patients were encouraged to resume normal activities and/or work as soon as possible.

\section{Assessment}

The duration of surgery, level of experience attained by the surgeon, and the identification and possible dissection of

Table 1 Patient characteristics

\begin{tabular}{lllll}
\hline & & Lichtenstein & Kugel & $p$ Value \\
\hline No. of patients & & 86 & 86 & \\
Mean (SD) age (years) & & $54.4(13.6)$ & $55.6(15.8)$ & .384 \\
Sex ratio (M:F) & & $85: 1$ & $85: 1$ & 1.00 \\
Mean (SD) BMI $\left(\mathrm{kg} / \mathrm{m}^{2}\right)$ & & $25.4(2.7)$ & $25.1(2.9)$ & .447 \\
ASA group & I & 59 & 54 & .657 \\
& II & 26 & 30 & \\
Employment & III & 1 & 2 & .315 \\
& Light & 37 & 27 & \\
& Heavy & 24 & 25 & .843 \\
Presence of hernia & None or retired & 20 & 26 & \\
& Missing & 5 & 8 & \\
& Weeks & 23 & 20 & \\
& Months & 43 & 45 & 13 \\
\hline
\end{tabular}

$B M I$ body mass index; $A S A$ American Society of Anesthesiologists; $S D$ standard deviation 
nerves and vessels in the operative field were recorded. In the diary the patients received, three sections were completed: the first section consisted of the study information and informed consent; the second section was devoted to recording height, weight, employment, and the duration of the hernia. The third part included were a preoperative visual analog scale (VAS) pain score and a questionnaire about the use of analgesics and the reasons for their consumption (pain due to the hernia, back pain, arthritis, headache, or other). The VAS pain score is a scale indicating a range of pain from zero, indicating no pain, to 100 , the least bearable pain, on a $100-\mathrm{mm}$ line. For calculation, the scores were rounded to the closest centimeter. Patients were asked to complete the second part preoperatively. At discharge patients took the diary home to complete the third section during the first 2 postoperative weeks. They recorded daily VAS pain scores as well as entries concerning the number and kind of analgesics taken. At 3 months after the operation all patients visited a surgeon for a physical and neurological exam. This evaluator was not the surgeon who had performed the operation. Any complications were recorded. Furthermore the patients completed a pain questionnaire. This included a VAS pain score and questions on the number and kind of analgesics used. Other questions were about numbness, bulging, and cutaneous sensory changes at the operative site. Bulging was defined as a bulge in the groin and demanded a physical examination (positive Valsalva test) or an ultrasound test to confirm any recurrence. For the sensory changes, a patient could choose from several sensory (neuropathic and nociceptive) and affective descriptors.

\section{Objectives}

The aim of the present study was to test the hypotheses that Kugel hernioplasty has less postoperative pain than the standard Lichtenstein repair, and that its feasibility is comparable. For the first hypothesis, the primary endpoint was the VAS pain score at 3 months. A score above zero at this time point is regarded as chronic pain as defined by the International Association for the Study of Pain [6]. Secondary outcome measures were pain scores and number of analgesics consumed during the first 2 weeks, as well as chronic pain descriptions. The duration of the procedure as well as number of complications were used for evaluating the second hypothesis.

\section{Statistical analysis}

According to the power calculations, 77 subjects per treatment group were needed for the study to achieve a statistical power of $90 \%$ with an alpha of $5 \%$. The calculations were made with a two-sided test for the VAS pain score at 3 months, considering a difference in VAS score of 1.0 as clinically significant. Based on a previous study, the standard deviation was set at 1.9 [7].

Statistical comparison was made with the Wilcoxon test for continuous variables. For ordinal data, the Pearson $\chi^{2}$ test was used; $p<.05$ was considered statistically significant. Data were analyzed on the basis of intention to treat.

\section{Results}

A total of 172 patients were randomly allocated to receive one of the two repairs. Two patients died during the study for reasons not related to the operation. Ultimately, 166 of 170 patients $(97.6 \%)$ were analyzed for the primary endpoint (Fig. 1). Baseline characteristics and pre-existent pain complaints were comparable between the groups (Tables 1 and 2, Fig. 2).

The mean VAS pain score was lower in the Kugel group than in the Lichtenstein group for every day of the first 2 postoperative weeks (Fig. 3). During the same period there were no significant differences in the total number of consumed paracetamol tablets (19.9 versus $18.5 ; p=.400)$ or in the number of consumed meloxicam tablets (8.6 versus $7.0 ; p=.295)$. On the days that the patients did not consume any analgesics (1,176 days), the mean VAS pain score was 1.4.

At 3 months, patients who underwent the open preperitoneal mesh repair reported significantly less pain (mean VAS score 0.3 versus $0.9, p=.002$ ). The number of

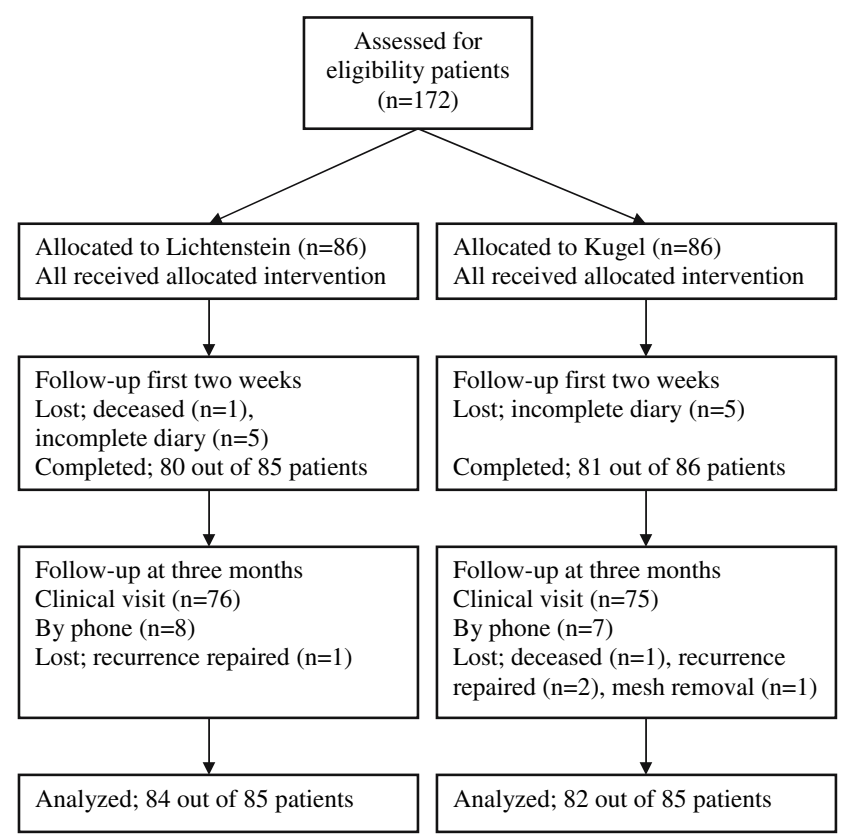

Fig. 1 Trial flow chart 
Table 2 Pre-existing pain complaints

\begin{tabular}{lllll}
\hline $\begin{array}{l}\text { Mean VAS } \\
\text { pain score (SD) }\end{array}$ & & $\begin{array}{l}\text { Lichtenstein } \\
(n=81)\end{array}$ & $\begin{array}{l}\text { Kugel } \\
(n=78)\end{array}$ & $p$ Value \\
\hline \multirow{2}{*}{ Use analgesics $(n)$} & $2.8(2.3)$ & $2.8(2.3)$ & .926 \\
& Sometimes & 6 & 10 & .952 \\
Reasons $(n)$ & Regular & 4 & 7 & \\
& Back pain & 2 & 5 & .505 \\
& Arthritis & 0 & 1 & \\
& Headache & 3 & 7 & \\
& Hernia & 5 & 4 & \\
\hline
\end{tabular}

VAS visual analog scale

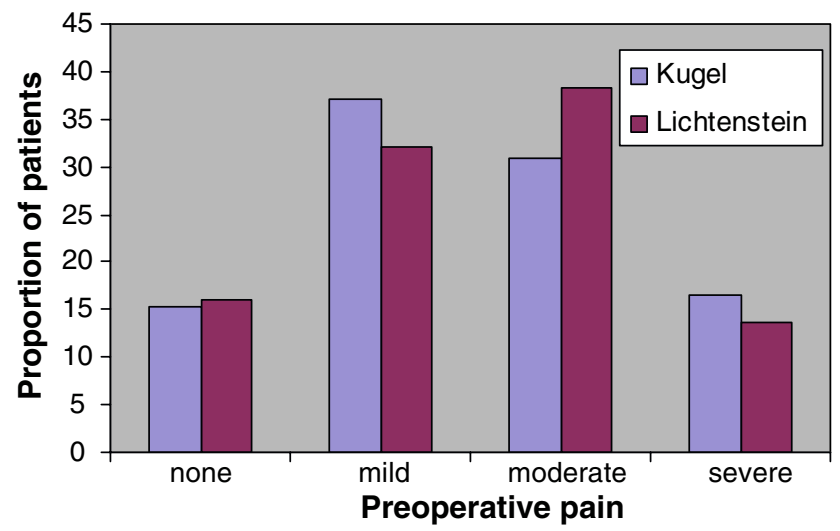

Fig. 2 Distribution of preoperative pain

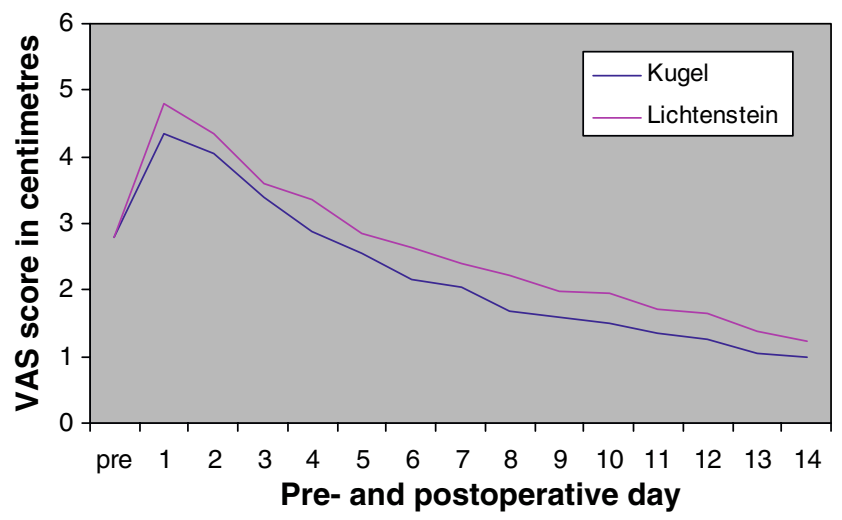

Fig. 3 Mean visual analog scale (VAS) pain score per day

patients reporting chronic pain was 17 of $82(20.7 \%)$ in the Kugel group and 34 of $84(40.5 \%)$ in the Lichtenstein group $(p=.007)$. In the 131 procedures performed by a first surgeon with a history of more than 5 procedures, 44 cases of chronic pain were recorded, an incidence not a significantly higher than that recorded with less experienced first surgeons ( 7 of $35, p=.122$ ). The pain intensity distribution is detailed in Figure 4. Eight patients who reported pain at 3 months used analgesics; paracetamol $(n=2)$, meloxicam

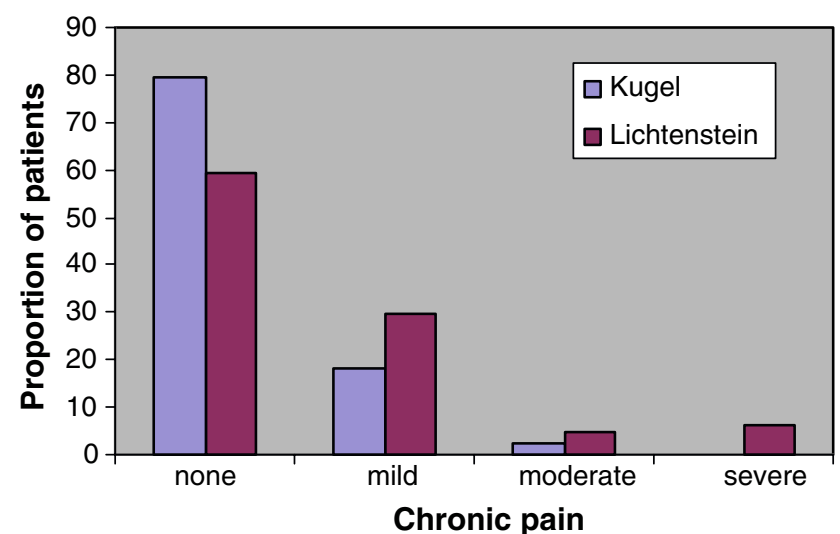

Fig. 4 Distribution of chronic pain

or ibuprofen $(n=3)$, and combinations $(n=3)$. Forty-one patients used nine different descriptors to describe their pain, mostly neuropathic (Table 3).

In the Lichtenstein group the operation took significantly longer ( 54 min versus $41 \mathrm{~min} ; p<.001$ ). The nerves were encountered significantly more frequently when the anterior approach was employed, and the inferior epigastric vessels were encountered more frequently with the preperitoneal approach (Table 4). Complications included hematoma, which was treated conservatively $(n=14)$; infection $(n=5)$; dysejaculation $(n=1)$ and urination problems resulting from pressure of the mesh against the bladder, as seen on a CT scan. There were no significant differences between the two groups with regard to recurrence. Diagnosed by either physical examination $(n=3)$ or ultrasound ( $n=1)$, each group had two recurrences. Three patients with recurrences underwent reoperation within 3 months. Another 12 Lichtenstein and 5 Kugel patients had a swelling/bulging recorded by physical examination or in the patient questionnaire. In no case was the bulging indicative of recurrence, as stated by the consulting physician or a confirming ultrasound test.

Numbness was reported significantly more in the Lichtenstein group (22 versus $3, p<.001)$. For patients with reported numbness, nerve damage was reported by the surgeon as the cause in 3 cases, and hypoesthesia was found on neurological examination in 15 cases. Cutaneous sensory changes were found in 6 Kugel patients and 24 Lichtenstein patients. The corresponding nerves were the iliohypogastric $(n=17)$, genitofemoral (femoral branch $n=6$ and genital branch $n=3)$, and ilioinguinal $(n=4)$. The sensory changes were related to the number of nerves encountered $(\mathrm{R}=.152, p=.050)$. The Pearson $\chi^{2}$ test for patients with a cutaneous sensory change of reported numbness was $37.4(p<.001)$; that of reported chronic pain, $18.3(p<.001)$. Patients without any sensory changes scored their pain as significantly less (.41 versus 1.53 , $p<.001)$ at 3 months. 
Table 3 Pain descriptors

\begin{tabular}{lll}
\hline & Lichtenstein $(n=28)$ & Kugel $(n=13)$ \\
\hline Neuropathic & & \\
Aching & 1 & 1 \\
Burning & 10 & 2 \\
Pricking & 17 & 5 \\
Shooting & 2 & 5 \\
Nociceptive & & \\
Gnawing & 0 & 1 \\
Pulling & 1 & 1 \\
Tender & 7 & 2 \\
Affective & & \\
Tiring & 2 & 0 \\
Irritating & 2 & 2 \\
\hline
\end{tabular}

Table 4 Procedure encounters

\begin{tabular}{llll}
\hline & $\begin{array}{l}\text { Lichtenstein } \\
(n=86)\end{array}$ & $\begin{array}{l}\text { Kugel } \\
(n=84)\end{array}$ & $p$ Value \\
\hline Ilioinguinal nerve & 67 & 9 & .000 \\
Damaged & 5 & 0 & .025 \\
Iliohypogastric nerve & 23 & 4 & .000 \\
Damaged & 3 & 1 & .325 \\
Genitofemoral nerve & 21 & 6 & .002 \\
Damaged & 1 & 0 & .323 \\
Inferior epigastric vessels & 42 & 73 & .000 \\
Vas deferens & 78 & 79 & .413 \\
\hline
\end{tabular}

For the entire group, the preoperative VAS pain score correlated with the scores in the first 2 weeks after the repair $(\mathrm{R}=.445, p<.001)$, the number of paracetamol taken $(\mathrm{R}=.260, p=.001)$, and the number of meloxicam taken $(\mathrm{R}=.205, p=.010)$. No significant correlation with the pain score was found at 3 months. The preoperative consumption of analgesics had no influence.

\section{Discussion}

In this randomized trial comparing the standard open anterior approach to hernia repair with an open preperitoneal approach, some significant advantages were found for the open preperitoneal repair technique. The procedure was performed in less time; at a 3 months follow-up, a lower mean VAS pain score and a smaller proportion of patients with chronic pain were reported than in the Lichtenstein group. The chronic pain was mostly described as neuropathic, because nerves were encountered more frequently with the anterior approach. In the open preperitoneal repair group less numbness was reported and fewer cutaneous sensory changes were found at neurological examination.

The authors regard chronic pain as a more severe complication than recurrence, because of its incapacitating character. Therefore the primary endpoint of this study was the mean VAS pain scores at 3 months. The VAS has it limitations, as it measures pain only one-dimensionally without regard to frequency. Nevertheless, this instrument is by far the most used measurement tool, and it is useful for comparisons like those made in the present study. The VAS pain score differed significantly between the groups, although the absolute difference was only 0.6. As stated in the Methods section, this might not be regarded as clinically relevant. Only four patients in each group used analgesics at this point. Thirty-four Lichtenstein patients $(40 \%)$, however, reported a VAS pain score higher than zero. This figure is relatively high compared to some other published data [8-10], although similar results have been reported from studies with comparable designs [7, 11, 12]. In the present study, the chronic pain of $40 \%$ in the Lichtenstein group is almost twice the rate found in the Kugel group (17 of 82 , or $21 \%$ ). The pain was merely described as "neuropathic," and it is assumed that this pain arises from nerve damage, which is more likely to occur with the Lichtenstein procedure because the ilioinguinal, iliohypogastric, and genitofemoral nerves are encountered significantly more frequently with this approach. The higher proportion of numbness and cutaneous changes underline this assumption.

Where some parameters are concerned, the procedures had similar outcomes. In the first 2 weeks postoperatively there were no clinically important differences in pain VAS score or in number of analgesics taken; nor were significant differences seen in the incidence or types of complications. One complication specific to the preperitoneal approach was seen in this study: higher urinary frequency (1 patient). A CT scan revealed impression of the mesh on the bladder, and removal of the preperitoneal mesh resolved the problem, and a definitive hernia repair is planned. In both groups two recurrences were found, and all four were regarded as technical failures. For some investigators, recurrence is of great concern. Schroder et al. reported a recurrence rate of $7.7 \%$ with the Kugel technique [13]. Because most failures occurred in their first 36 cases, they attribute this to the learning curve. They summarize other studies wherein preperitoneal repairs are noted for steep learning curves. In contrast, acceptable recurrence rates have been reported in numerous studies with long-term follow-up, with the recurrence rate varying from $0 \%$ to $0.8 \%[4,14-17]$. Nevertheless, we have the impression that the preperitoneal approach is more technically demanding, probably because the preperitoneal space is more unknown. We therefore established learning workshops before this 
study was begun. In addition, patients with recurrent hernias and large direct hernias were excluded. The experience of the operating surgeon was dichotomized into five or fewer procedures and more than five procedures, similar to the design of a previous study from the same institution to collect comparable data [18]. In practice, the experience of the supervisor exceeded 20 procedures. The level of experience of the supervising surgeon was not specified, and it is therefore possible that a procedure carried out under less experienced supervision might have failed, thereby contributing to the recurrence rate of $2.3 \%$ (4 of 172).

The Kugel patch is heavier than a monofilament Prolene mesh. It is double layered with a memory coil [4]. Thus it might be assumed that this patch is stiffer, resulting in more pain [11]. At the 3-month follow-up, however, significantly less pain and less swelling/bulging was found in the Kugel group than in the Lichtenstein group.

Only one previous study known to the authors has compared the two hernia repair techniques, that by Dofru et al. [16], who found similar results with regard to operating time and complications. However, no pain-related outcomes were included. The present study has its own limitations: (1) The Kugel procedure requires a more cranial incision, and knowledge of this feature impairs the blindness of the patient and the evaluator. However, none of our patients realized this when they were seen at followup. (2) Fifteen of our patients did not have a physical or neurological follow-up examination, because they had passed the clinical visit and thereafter would only be contacted by telephone. These 15 patients were more or less equally divided between the two groups, and none of them reported any pain at the follow-up interview. The pain-related outcomes are therefore at least not overestimated. (3) The use of a VAS pain score as the primary endpoint has limitations, because pain is far more than a one-dimensional score. Additional pain parameters had to be employed to compensate for this. Nevertheless, it is difficult to express the significance of every VAS pain score higher than zero. In half of the immediate postoperative period (1,176 of 2,406 days) patients reported a pain score without taking any pain medication, as did 43 of 51 patients reporting pain at 3 months. This occurred even though the surgeons had advised patients concerning the standard pain protocol and explained the use of analgesics to the patients.

Within the limitations of this study, an open preperitoneal repair like the Kugel repair has shown some clinically relevant advantages. As the learning curve flattens, this repair may also be suitable in the treatment of large direct and recurrent hernias. For surgeons who prefer an open approach, the Kugel procedure is a feasible alternative for the standard Lichtenstein procedure and is associated with less chronic pain at 3 months, most likely because fewer nerves are at risk than with the anterior approach. The superiority of the approach will demonstrated if education of surgeons focuses on the preperitoneal space as it has emphasized the inguinal canal.

\section{References}

1. Douek M, Smith G, Oshowo A, et al. (2003) Prospective randomised controlled trial of laparoscopic versus open inguinal hernia mesh repair: five year follow up. BMJ 326:1012-1013

2. Grant AM, Scott NW, O'Dwyer PJ, MRC Laparoscopic Groin Hernia Trial Group (2004) Five-year follow-up of a randomized trial to assess pain and numbness after laparoscopic or open repair of groin hernia. Br J Surg 91:1570-1574

3. MRC Laparoscopic Groin Hernia Trial Group (1999) Laparoscopic versus open repair of groin hernia: a randomised comparison. Lancet 354:185-190

4. Kugel RD (1999) Minimally invasive, nonlaparoscopic, preperitoneal, and sutureless, inguinal herniorrhaphy. Am J Surg 178:298-302

5. Amid PK (2004) Lichtenstein tension-free hernioplasty: its inception, evolution, and principles. Hernia 8:1-7

6. Classification of chronic pain: descriptions of chronic pain syndromes, definitions of pain terms (1994) In Merskey H, Bogduk N, editors, Task Force on Taxonomy of the IASP, 2nd Edition, Seattle, WA, IASP Press 209-214

7. Nienhuijs SW, van Oort I, Keemers-Gels ME, et al. (2005) Randomized trial comparing the Prolene hernia system, mesh plug repair and Lichtenstein method for open inguinal hernia repair. Br J Surg 92:33-38

8. Bringman S, Ramel S, Heikkinen TJ, et al. (2003) Tension-free inguinal hernia repair: TEP versus mesh-plug versus Lichtenstein: a prospective randomized controlled trial. Ann Surg 237:142-147

9. Moreno-Egea A, Martinez JAT, Cuenca GM, et al. (2004) Randomized clinical trial of fixation vs nonfixation of mesh in total extraperitoneal inguinal hernioplasty. Arch Surg 139:1376-1379

10. Ravichandran D, Kalambe BG, Pain JA (2000) Pilot randomized controlled study of preservation or division of ilioinguinal nerve in open mesh repair of inguinal hernia. Br J Surg 87:1166-1167

11. O'Dwyer PJ, Kingsnorth AN, Molloy RG, et al. (2005) Randomized clinical trial assessing impact of a lightweight or heavyweight mesh on chronic pain after inguinal hernia repair. $\mathrm{Br}$ J Surg 92:166-170

12. Nienhuijs SW, Remijn EE, Rosman C (2005) Hernia repair in elderly patients under unmonitored local anaesthesia is feasible. Hernia 9:218-222

13. Schroder DM, Lloyd LR, Boccaccio JE, et al. (2004) Inguinal hernia recurrence following preperitoneal Kugel patch repair. Am Surg 70:132-136

14. Ceriani V, Faleschini E, Bignami P, et al. (2005) Kugel hernia repair: open "mini-invasive" technique. Personal experience on 620 patients. Hernia 9:344-347

15. Fenoglio ME, Bermas HR, Haun WE, et al. (2005) Inguinal hernia repair: results using an open preperitoneal approach. Hernia 9:160-161

16. Dogru O, Girgin M, Bulbuller N, et al. (2006) Comparison of Kugel and Lichtenstein operations for inguinal hernia repair: results of a prospective randomized study. World J Surg 30:346350 
17. Misawa T, Sakurai M, Kanai H, et al. (2005) Kugel herniorrhaphy: clinical results of 124 consecutive operations. Surg Today 35:639-644
18. Nienhuijs S, Kortmann B, Boerma M, et al. (2004) Preferred mesh-based inguinal hernia repair in a teaching setting. Results of a randomized study. Arch Surg 139:1097-1100 